\title{
Augmented upper body contribution to oxygen uptake during upper body exercise with concurrent leg functional electrical stimulation in persons with spinal cord injury
}

\author{
WT Phillips and LN Burkett \\ Department of Exercise Science and Physical Education, Arizona State University, Tempe, Arizona 85287-0701, USA
}

\begin{abstract}
The purpose of this pilot study was to compare the contribution of upper body musculature to $\mathrm{VO}_{2}$ with and without concurrent leg FES (LFES). Eight subjects with spinal cord injury, lesion levels range $\mathrm{C} 6$ - T12, performed upper body exercise (UBE) during no LFES (NOS), LFES at $40 \mathrm{~mA}$ (LOS), and $80 \mathrm{~mA}$ (HIS), at rest, $60 \%$ and $80 \%$ of $\mathrm{VO}_{2 \text { peak }}$. Resting $\mathrm{VO}_{2}$ values were obtained during NOS, LOS and HIS conditions and were then subtracted from their respective whole body $\mathrm{VO}_{2}$ values to give an estimate of upper body $\mathrm{VO}_{2}$. Small and non significant increases were found in the HIS vs NOS condition at $60 \% \mathrm{VO}_{2 \text { peak }}$. Larger differences of $7.8 \%$ were found in the HIS vs NOS condition at $80 \% \quad \mathrm{VO}_{2 \text { peak }}$ $\left(11.35 \pm 3.8 \mathrm{ml} \mathrm{kg}^{-1} \mathrm{~min}^{-1}\right.$ to $\left.12.24 \pm 4.0 \mathrm{ml} \mathrm{kg} \mathrm{min}^{-1}\right)$, although this too was not significant, perhaps due to the small number of subjects in this study and the consequently low statistical power to detect a significant difference. We discuss the implications for these preliminary results in the context of the existing literature on this topic.
\end{abstract}

Keywords: electrical stimulation; arm cranking; CHD risk; quadriplegic; paraplegic

\section{Introduction}

Coronary heart disease (CHD) is now one of the leading causes of death for persons with a spinal cord injury (SCI). ${ }^{1,2}$ Major contributors to CHD risk are low levels of daily activity, ${ }^{3,4}$ low aerobic fitness ${ }^{5,6}$ low muscular endurance, and an inability to achieve high levels of oxygen uptake $\left(\mathrm{VO}_{2}\right),{ }^{7-9}$ which has been reported as primarily due to a markedly diminished venous return secondary to paralysis. ${ }^{10-12}$ Persons with SCI are also reported as suffering from abnormally low levels of high density lipoprotein (HDLc), perhaps as a result of these low activity levels. ${ }^{1,3,13,14}$ Functional electrical stimulation of the lower limbs (LFES) in conjunction with upper body exericse (UBE) improves acute responses for both $\mathrm{VO}_{2}{ }^{10,15}$ and venous return ${ }^{16,17}$ when compared to UBE alone. The question remains as to whether the increased body $\mathrm{VO}_{2}$ is due entirely to the greater muscle mass being activated via LFES, or whether there is an augmented contribution from the upper body musculature secondary to an LFES-enhanced upper body flow as has been suggested by some authors. ${ }^{7}, 18$ Glaser et al. ${ }^{10}$ have reported increases in $\mathrm{VO}_{2 \max }$ and lower perceived exertion (RPE) scores during UBE with concurrent LFES compared to UBE alone in a group of $10 \mathrm{SCI}$ individuals. Thomas et al. ${ }^{19}$ have also reported FESinduced increases in $\mathrm{VO}_{2 \max }$ during concurrent wheel-

Correspondence: Wayne T Phillips, Department of Exercise Science and Physical Education, Arizona State University, Tempe, Arizona 85287-0701, USA chair propulsion. Although these two latter studies would seem to suggest an LFES-enhanced contribution to upper body $\mathrm{VO}_{2}$, no discussion of causal mechanisms was reported. In contrast to this, other authors have reported no differences in RPE, ${ }^{12,15,19}$ or maximal $\mathrm{VO}_{2}{ }^{12,20,21}$ during UBE with concurrent LFES. No studies could be found which have specifically addressed issues of upper body hemodynamics during UBE with concurrent LFES in persons with SCI. The purpose of this pilot study is to obtain preliminary data on this issue by re-analyzing data obtained from a previous investigation. ${ }^{15}$ An LFES-augmented upper body contribution to $\mathrm{VO}_{2}$ would indicate an augmented upper body blood flow secondary to the LFES. This in turn may reduce the perception of fatigue, improve responses to conventional UBE training and thus contribute to lower CHD risk.

\section{Methods}

Eight healthy subjects with spinal cord injury (mean age $33 \pm 8$, range 21-45 years) and lesion levels between C6 and T12 volunteered for this study. Seven of the subjects were motor complete, one was motor incomplete, and two of the subjects reported some degree of sensation in the lower limbs. Subjects characteristics appear in Table 1. All subjects were made fully conversant with the study protocol via individualized familiarization periods for both UBE and FES equipment, conducted over $2-3$ weeks. This 
period also served to assess their safe and appropriate responses to the treatment. Exclusion criteria for selection were: inability to elicit visible muscular contraction during LFES; excessive spasticity and/or currently taking medication for spasticity; over sensitivity to LFES stimulation; symptoms of autonomic dysreflexia, or any other condition determined by their physician to be detrimentally effected by the LFES. No subjects suffered from contractures, though all reported occasional muscular spasms, and none reported any history of sensitivity to heat. Subjects were cleared by their physician for participation in this study, received detailed verbal and written descriptions of the experimental procedures, and completed informed consent in accordance with the Human Subjects Institutional Review Board. All subjects were recreationally active, and continued to be so both before and during the study period. Subjects were asked to avoid vigorous exercise for the $24 \mathrm{~h}$ preceding each exercise session, and to refrain from eating for at least $4 \mathrm{~h}$ before testing. In addition subjects were asked to ensure that their bowel and bladder program was performed before attending each session.

Details of testing procedures have been reported previously, ${ }^{15}$ but, briefly, consisted of an initial maximal UBE test without LFES, and then three identical submaximal tests each of which required subjects to work at $60 \%$ and $80 \%$ of a previously determined $\mathrm{VO}_{2 \text { peak }}$. Each submaximal session was performed with either no LFES (NOS), LFES at $40 \mathrm{~mA}$ (LOS), or $80 \mathrm{~mA}$ (HIS). Stimulation was applied to both lower limbs, with two EMPI 'Focus' Neuromuscular Stimulators (EMPI Inc. St Paul, Minnesota), over the motor points of each major muscle group (quadriceps, hamstrings gastrocnemius and tibialis anterior). Stimulation consisted of symmetric biphasic pulses of $300 \mathrm{~ms}$ at $35 \mathrm{~Hz}$ delivered across a 1000-ohm load, over a 'duty cycle' of $2.5 \mathrm{~s}$ 'on' and $5 \mathrm{~s}$ 'off', with a $2 \mathrm{~s}$ 'rise time' and a $2 \mathrm{~s}$ 'fall time' for each pulse. Upper and lower leg muscles were stimulated alternately, with the stimulation 'pattern' set so that the 'fall time' of the upper leg muscles coincided with the 'rise time' of the lower leg muscles, and vice versa. The three stimulation conditions were randomly applied across exercise sessions and all tests were separated by at least $24 \mathrm{~h}$.

Metabolic indices were monitored continuously by open-circuit spirometry, and averaged over $30 \mathrm{~s}$ intervals using a Horizon Metabolic Cart (Sensormedics Corporation, Yorba Linda, CA). Blood pressure (BP) was monitored by auscultation during the final minute of each stage of the $\mathrm{VO}_{2 \text { peak }}$ test, and at the end of every third minute during the sub maximum tests. Measures were taken on the right arm, during the exercise session, while one of the researchers assisted the subject in maintaining the arm crank cadence. This technique resulted in an inconsistent work output by most subjects during BP measurement, due to the amount of assistance required to maintain single-arm cadence. The authors decided therefore,
Table 1 Subject characteristics

\begin{tabular}{lcccccc}
\hline Subject & Age & Sex & Wt $(\mathrm{kg})$ & Lesion & Com $/$ inc & Yrs $P-I$ \\
\hline 1 & 36 & M & 68.1 & C6/7 & com & 1.3 \\
2 & 36 & M & 72.3 & C8/T1 & inc & 8.5 \\
3 & 45 & F & 65.9 & C8/T1 & com & 14.0 \\
4 & 21 & M & 59.1 & T4/T7 & com & 1.0 \\
5 & 25 & M & 61.4 & T5 & com & 8.5 \\
6 & 36 & M & 75.0 & T8 & com & 1.6 \\
7 & 30 & M & 84.5 & T10 & com & 6.0 \\
8 & 36 & M & 65.5 & T12 & com & 3.0 \\
Means & $33 \pm 8$ & & $69 \pm 8$ & & & $6 \pm 4$ \\
\hline
\end{tabular}

Note: All means $\pm \mathrm{SD}$, rounded to nearest whole number. Key: 'Level' = level of spinal lesion: 'Yrs P-I' = number of years post injury: 'Com' = complete lesion: 'Inc' $=$ incomplete lesion

that blood pressure readings could not be regarded as valid data for analysis but continued to use them as a safety monitoring procedure. Heart rate (HR) was constantly monitored by a Polar 'Vantage XL' Heart Rate Monitor (Polar, USA, Inc, Stamford, CT).

\section{Estimation of upper body contribution to $\mathrm{VO}_{2}$}

In our original study, each of the three stimulation conditions included a 3-4 min pre-exercise resting period during which $\mathrm{VO}_{2}$ was measured while subjects sat quietly with hands resting on the ergometer handles. This gave three separate 'resting' $\mathrm{VO}_{2}$ values for all subjects, (i) a 'true' resting value, (ie with no LFES applied), (ii) a 'LOS resting' value (ie rest during LFES applied at $40 \mathrm{~mA}$ ) and (iii) a 'HIS resting' value (ie rest during LFES applied at $80 \mathrm{~mA}$ ). Whole body $\mathrm{VO}_{2}$ may be considered to be made up of the total of resting, LFES and UBE components according to the following equation.

$$
\begin{aligned}
& \text { Whole body } \mathrm{VO}_{2} \\
& =\left(\text { resting } \mathrm{VO}_{2}\right. \\
& \left.+ \text { LFES generated } \mathrm{VO}_{2}\right) \\
& + \text { UBE generated } \mathrm{VO}_{2}
\end{aligned}
$$

Subtracting the resting $\mathrm{VO}_{2}$ (with or without LFES) for each condition from its corresponding whole body value controls for the additional $\mathrm{VO}_{2}$ generated by the LFES-activated muscle mass and gives an estimate of oxygen uptake for the upper body alone.

$$
\begin{aligned}
& \text { UBE generated } \mathrm{VO}_{2}=\text { Whole body } \mathrm{VO}_{2} \\
& -\left(\text { resting } \mathrm{VO}_{2}+\text { LFES generated } \mathrm{VO}_{2}\right)
\end{aligned}
$$

If the increase in whole body $\mathrm{VO}_{2}$ during the LFES condition is solely a result of the LFES-activated muscle mass then the 'separated out' contribution of the upper body musculature ('UBE generated $\mathrm{VO}_{2}$ ') will be the same during all three exercise conditions. If however the increased $\mathrm{VO}_{2}$ is due in part to an LFESaugmented upper body blood flow, then the UBE generated $\mathrm{VO}_{2}$ will be greater during the LFES conditions than the non-LFES condition. 
Analysis

Overall differences for both whole body and UBEgenerated $\mathrm{VO}_{2}$ were analyzed with a repeated measures ANOVA. Differences between factor levels were determined with Scheffe's post hoc test. All differences were considered statistically significant at $P<0.05$.

\section{Results}

Whole body $\mathrm{VO}_{2}$ increased significantly between stimulation conditions (Figure 1a), the contribution of 'resting' $\mathrm{VO}_{2}$ for each condition being indicated by the black bars. Upper body contribution to $\mathrm{VO}_{2}$ increased by $2.5 \%$ between NOS and HIS at $60 \%$ $\mathrm{VO}_{2 \text { peak }}\left(8.52 \pm 1.9 \mathrm{ml} \mathrm{kg}^{-1} \mathrm{~min}^{-1}\right.$ to $8.74 \pm 2.3 \mathrm{ml}$ $\mathrm{kg}^{-1}$ min $^{-1}$, ns) and by $7.8 \%$ between NOS and HIS at $80 \% \mathrm{VO}_{2 \text { peak }}\left(11.35 \pm 3.8 \mathrm{ml} \mathrm{kg}^{-1} \mathrm{~min}^{-1}\right.$ to $12.24 \pm$ $4.0 \mathrm{ml} \mathrm{kg}^{-1} \min ^{-1}$, ns) (Figure 1b).

\section{Discussion}

Previous authors investigating blood flow changes in persons with a SCI have suggested that the application of LFES may make more blood available to the upper body musculature during UBE, and thus contribute to an increase in whole body $\mathrm{VO}_{2} \cdot{ }^{22-24}$ Such mechanisms remain unconfirmed however since few studies have directly investigated upper body hemodynamics in persons with a spinal cord injury, the majority focusing predominantly on central hemodynamic changes secondary to lower limb blood pooling. ${ }^{10,12,25,26}$ Glaser $^{27}$ and Figoni ${ }^{28}$ have reported the separate and relative contributions of resting, LFES, and voluntary arm exercise (UBE) conditions to whole body $\mathrm{VO}_{2}$ in persons with spinal injuries at levels $\mathrm{T} 8$ and $\mathrm{C} 7$ respectively, and have noted the additive effect of these three sources of energy expenditure. Our study is the first we are aware of to directly address the potential upper body augmentative benefits of LFES in a group of persons with SCI. Specifically we wished to determine whether the increased whole body $\mathrm{VO}_{2}$ during UBE with concurrent LFES reported in our previous study ${ }^{15}$ was entirely due to the lower limb muscle mass activated by the LFES, or whether a portion of the increased whole body $\mathrm{VO}_{2}$ could be attributed to an augmentation of the upper body contribution secondary to the LFES. In the current study, using identical UBE protocols, we found a mean increase of $7.8 \%$ in the upper body contribution to $\mathrm{VO}_{2}$ during the HIS condition compared to the NOS (a) Whole body $\mathrm{VO}_{2}$

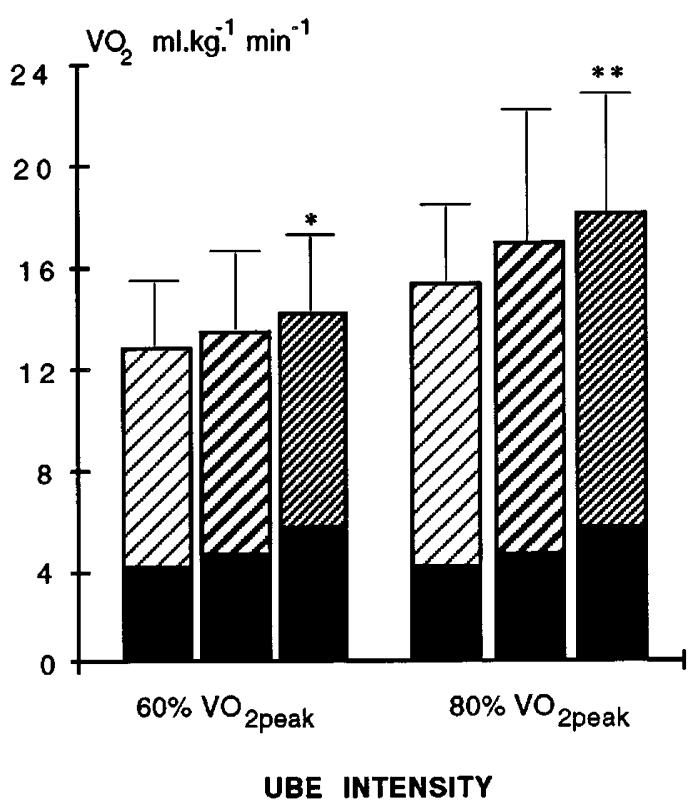

(b) Upper body $\mathrm{VO}_{2}$

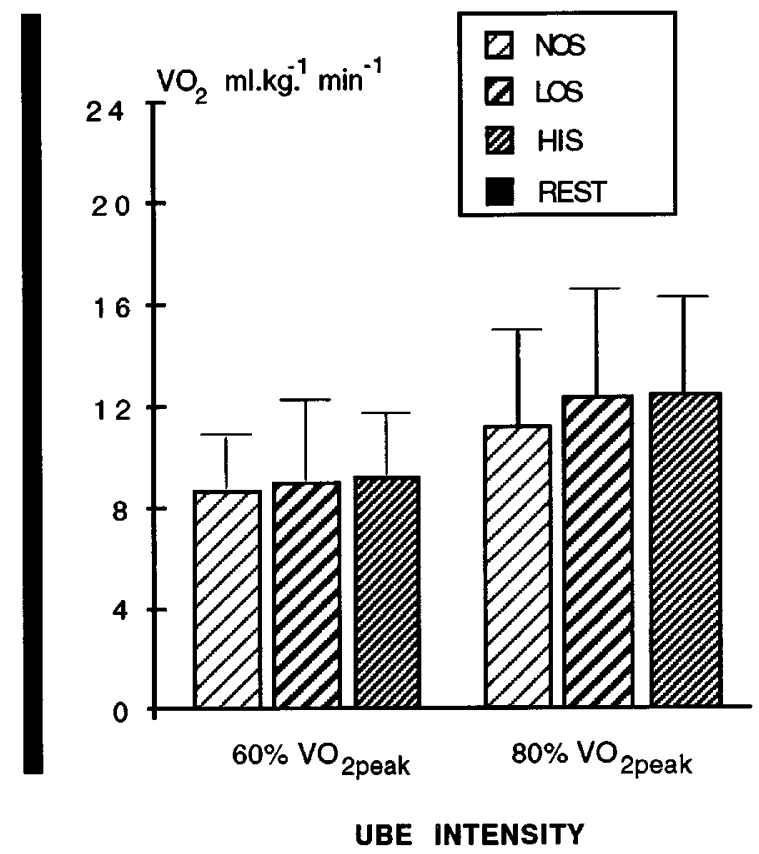

Note: Means \pm standard deviation

* $\quad=$ Significantly different from NOS at same UBE intensity $p \leq .05$

$* * \quad=$ Significantly different from NOS at same UBE intensity $p \leq .01$

Figure 1 
condition at $80 \% \mathrm{VO}_{2 \text { peak }}$ (Figure $1 \mathrm{~b}$ ). Although this result was not statistically significant there are a number of reasons why this finding is of interest.

Glaser et al. ${ }^{10}$ using a protocol similar to ours reported a significant LFES-induced increase in submaximal $\mathrm{VO}_{2}$ and a significant $8.7 \%$ LFESinduced increase in maximal $\mathrm{VO}_{2}$. Thomas et al. ${ }^{19}$ have also reported significant increases in submaximal $\mathrm{UBE}$ at $68 \% \mathrm{VO}_{2 \text { peak }}(10.6 \%$ to $12 \%)$, submaximal wheelchair propulsion $(5.7 \%$ to $22.8 \%$ ), and maximal wheelchair propulsion $(2 \%)$ with concurrent LFES compared to a non-LFES condition. Both these studies could be interpreted as indicating an augmented effect on upper body musculature although no discussion of this effect was reported. Our study findings of an augmented $7.8 \%$ increase in $\mathrm{VO}_{2}$ compares favorably with these results. Additionally the Glaser et al. ${ }^{10}$ study which used a similar protocol and stimulation pattern to ours would be expected to report higher differences since they utilized (i) a much higher intensity of stimulation (150 mA vs $80 \mathrm{~mA}$ ), and (ii) maximal UBE workloads compared to our submaximal intensity (at $80 \% \mathrm{VO}_{2 \max }$ ). Additionally our study was conducted with fewer subjects $(n=8 v s$ 10) than the Glaser et al. ${ }^{10}$ study and so would have less statistical power to detect a significant difference between conditions. No stimulation level or patterning is reported for the Thomas et al. ${ }^{19}$ study and so comparisons with their UBE protocol are impossible. In consideration of these data therefore we believe that our results, though not statistically significant, may be considered to be supportive of the concept of an LFES-induced augmentative effect on UBE in persons with a spinal cord injury.

Although no studies relating to the SCI population have directly addressed the augmentative effects of LFES on UBE, two recent studies ${ }^{29,30}$ with non-spinal cord injured subjects have investigated this issue. Eijsbouts et al. ${ }^{30}$ reported that the increased $\mathrm{VO}_{2 \max }$ found in their study during UBE with concurrent LFES compared to non LFES was a function solely of the amount of muscle mass engaged in the exercise - ie the $\mathrm{VO}_{2}$ increased in accordance with the additional lower limb muscle mass stimulated by the LFES. However although this well designed study provides important information on some of the mechanistic aspects of LFES augmentation of UBE, and is in apparent contrast to the studies of spinal cord injured subjects cited above, there are a number of factors which make such comparisons very difficult. All subjects in the Eijsbouts et al. ${ }^{30}$ study were able bodied - and so would be sensitive to the discomfort and potential pain of LFES administration. In this sense their responses would be totally unlike that of the majority of SCI individuals. If lower limb muscles were intended to be stimulated 'passively' as suggested by these authors, the amount and duration of muscle mass able to be impacted would vary considerably according to the discomfort and/or pain 'threshold' of the able bodied participant. Such variation could occur both between and within subjects and between and within sessions. Additionally, we have found that it is extremely difficult to obtain a 'passive' response from the lower limbs of an able bodied person during a progressive UBE test with the body in a conventional seated position since. In an able bodied person when the feet are placed on the floor during a seated UBE test, the muscles of the legs will invariably be used with ever increasing intensity to 'brace' the body in order to maintain stability as the UBE intensity is increased. In agreement with the findings of Eijsbouts et al. ${ }^{30}$ we also reported no increases in $\mathrm{VO}_{2 \max }$ between UBE and UBE with concurrent LFES in a group of able bodied subjects. ${ }^{29}$ In contrast to the Eijsbouts et $a .^{30}$ study however we concluded that any potential effect of the LFES was overshadowed by (i) the low levels of stimulation able to be voluntarily tolerated by most subjects (less than $40 \mathrm{~mA}$ in some cases), and (ii) the magnitude of the voluntary muscular contractions used for a 'bracing' effect from the lower limbs at higher intensities of UBE which in most cases was visibly greater than the contractile force generated by the LFES. ${ }^{29}$ Until methods can be designed to overcome such obstacles in able bodied populations, research to elucidate any augmentative effects of LFES on UBE will need to focus on persons with a spinal cord injury.

Differentiating upper and lower body contributions to $\mathrm{VO}_{2}$ during UBE with concurrent LFES will be important in elucidating the physiological mechanisms of exercise training-induced improvements in persons with a spinal cord injury. This is an area which has received little attention in the literature, although there are some indications that such protocols could have a beneficial effect on cardiovascular risk factors in the spinal cord injured population. Chronic increases in blood flow have been reported to increase the diameter of large blood vessels $\mathrm{s}^{31,32}$ and Shenberger et al..$^{33}$ have reported greater acute vasodilator capacity and larger brachial arteries in the forearms of active subjects with paraplegia compared to normal able-bodied controls, the authors suggesting that these differences were physical activity mediated. While similar effects could be elicited in the SCI population with conventional UBE training programs, the potential effects of LFES on upper body performance suggested by others $7,18,22-24$ and supported by the preliminary results of this study could perhaps augment these training benefits via an increased upper body blood flow secondary to the application of LFES. This in turn could result in an enhanced upper body $\mathrm{O}_{2}$ perfusion and extraction, and a peripheral training effect 'boost' to the voluntary musculature, increasing endurance capacity and/or the ability to generate higher levels of $\mathrm{VO}_{2}$. In support of this hypothesis, lower ratings of perceived exertion scores have been reported by one study during UBE with concurrent LFES compared to UBE alone ${ }^{10}$ and such reduced perceptions of fatigue may contribute to an increase in endurance performance. As has also been suggested by 
other authors, ${ }^{7,22-25}$ these effects could provide a platform for augmented fitness training with resultant beneficial effects on cardiovascular risk factors.

While such mechanisms remain speculative with only indirect and/or preliminary data available, the potential importance of this protocol merits further study particularly in the light of Objective 9.11 of Healthy People 2000, ${ }^{34}$ and recent recommendations from the Centers for Disease Control. ${ }^{35}$ These reports encourage the development and implementation of strategies and techniques which may prevent or ameliorate secondary disabilities in persons with a spinal cord injury. Our investigations thus align well with such objectives and for the reasons we have stated above, the results of our preliminary study points the way for more direct investigations of LFESinfluenced upper body hemodynamics in persons with a SCI. Even the relatively small augmentative effects of LFES reported by some authors ${ }^{10,19}$ and supported by the findings of the current study could nevertheless be an important contribution to the reduction of cardiovascular risk factors in a population with such chronically reduced capacity for maintaining and improving even low levels of aerobic fitness.

\section{Summary and conclusions}

In able bodied persons, physiological and hemodynamic responses to exercise are well established, responses to the frequency, intensity and duration of exercise training are generally predictable, and their effects on risk factor status has been the subject of considerable research. For persons with a spinal cord injury, such issues have been little addressed, particularly when used in conjunction with LFES. If LFES exercise training programs are to be developed to maximize improvements in cardiovascular health, it will be necessary to clarify the mechanisms of such improvements. This pilot study has focused on the potential contribution of UBE to whole body $\mathrm{VO}_{2}$ both with and without concurrent LFES. An LFESenhanced contribution by the upper body musculature to whole body $\mathrm{VO}_{2}$ may contribute to increased upper body endurance performance secondary to an enhanced upper body blood flow and reduced perception of fatigue. It may also augment (or 'boost') chronic training responses to the voluntary musculature. While such mechanisms and responses still remain speculative, our preliminary findings are supportive of the small number of studies which have indirectly reported the potential for an augmented upper body contribution to whole body $\mathrm{VO}_{2}$ secondary to concurrent LFES. Even the relatively small augmentation of $\mathrm{VO}_{2}$ which are suggested by us and these other studies could be important in reducing cardiovascular risk factors in a population with a dramatically reduced capacity for maintaining and improving aerobic fitness. Recent reports from federal institutions have encouraged the development and implementation of strategies and techniques to improve the health status of persons with a spinal cord injury. Our investigation aligns well with such objectives and our preliminary results, in conjunction with the reports of other authors point the way for a more in depth investigation of LFESinfluenced upper body hemodynamics in persons with a SCI. Further investigation of the responses and putative mechanisms highlighted in this pilot study should be conducted with greater numbers of subjects, higher intensities of LFES and more direct hemodynamic measures using plethysmographic techniques.

\section{Acknowledgements}

Thanks are extended to EMPI Incorporated for provision of the 'Focus' neuromuscular stimulators used in this study and supplies of electrodes.

\section{References}

1 Bauman WA, Spungen AM, Zhong Y-G, Rothstein JL. Depressed serum high density lipoprotein cholesterol levels in veterans with spinal cord injury. Paraplegia 1992; 30: 697-703.

2 DeVivo MJ, Black KJ, Stover SS. Causes of death during the first 12 years after spinal cord injury. Archives of Physical Medicine and Rehabilitation 1993; 74: $248-254$.

3 Hjeltnes N, Jansen T. Physical endurance capacity, functional status and medical complications in spinal cord injured subjects with long-standing lesions. Paraplegia 1990; 28: 428-432.

4 Kennedy DW, Smith RW. A comparison of past and future leisure activity participation between spinal cord injured and non-disabled persons. Paraplegia 1990; 28: 130-136.

5 LaPorte RE et al. The spectrum of physical activity, cardiovascular disease and health: An epidemiological perspective. American Journal of Epidemiology 1984; 120: 507-517.

6 Dearwater SR et al. Activity in the spinal cord-injured patient: An epidemiologic analysis of metabolic parameters. Medicine and Science in Sports and Exercise 1986; 18: $541-544$.

7 Glaser RM. Arm exercise training for wheelchair users. Medicine and Science in Sports and Exercise 1989; 21(suppl.): S149-S157.

8 Burkett LN, Chisum J, Stone W, Fernhall B. Exercise capacity of untrained spinal cord injured individuals and the relationship of peak oxygen uptake to level of injury. Paraplegia 1990; 28: $512-$ 521

9 Van Loan MD, McCluer S, Loftin JM, Bouleau RA. Comparison of physiological responses to maximal arm exercise among ablebodied, paraplegics and quadriplegics. Paraplegia; 1987; 25: $397-405$.

10 Glaser RM et al. Use of lower-limb FNS to improve arm exercise performance of SCI individuals. In: Proceedings of the 16th Annual RESNA Conference, pp. 416-418, 1993.

11 Figoni SF et al. FNS-assisted venous return in exercising SCI men. In: Proceedings of the International Conference of the Association of Advanced Rehabilitation Technology, Montreal, Canada, pp. 328-329, 1988.

12 Davis GM et al. Cardiovascular responses to arm cranking and FNS-induced leg exercise in paraplegics. Journal of Applied Physiology 1990; 69: 671-677.

13 Bauman WA, Alexander LR, Zhong Y-G, Spungen AM. Simulated leg ergometry training improves body composition and HDL-cholesterol values. In: American Paraplegia Society, 40th Annual Conference, Las Vegas, NV. 1994, p. 17.

14 LaPorte RE et al. HDL cholesterol across a spectrum of physical activity from quadriplegia to marathon running. The Lancet 1983; (May 28): 1212-1213.

15 Philips WT, Burkett LN. Arm crank exercise with static FNS in spinal cord injured persons. Medicine and Science in Sports and Exercise 1995; 27: pp. $530-535$. 
16 Philips WT et al. Relative changes in blood flow with functional electrical stimulation during exercise of the paralyzed lower limbs. Paraplegia 1995; 33: $90-93$.

17 Figoni SF, Glaser RM, Rogers MM. Hemodynamic responses of quadriplegics to arm, ES-leg, and combined arm and ES-leg ergometry. Medicine and Science in Sports and Exercise 1989; 21 (suppl.): S96.

18 Davis GM et al. Cardiovascular responses to FNS-induced isometric leg exercise during orthostatic stress in paraplegics. In: Proceedings of the International Conference of the Association for the Advancement of Rehabilitation Technology, Montreal, Canada 1988, pp. $95-96$.

19 Thomas AJ, Davis GM, Gass GC. Arm crank exercise with static FNS in spinal cord injured persons. Medicine and Science in Sports and Exercise 1992; 24 (suppl.): S18.

20 Climstein $\mathrm{M}$ et al. Electrical stimulation-induced leg exercise during arm cranking in paraplegic and able-bodied males. Medicine and Science in Sports and Exercise 1994; 26 (Abstr): S30.

21 Figoni SF et al. Physiologic responses of paraplegics and quadriplegics to passive and active leg cycle ergometry. Journal of the American Paraplegic Society 1990; 13: 33-39.

22 Davis GM. Exercise capacity of individuals with paraplegia. Medicine and Science in Sports and Exercise 1993; 25: 423-432.

23 Cowell LL, Squires WG, Raven PB. Benefits of aerobic exercise for the paraplegic: A brief review. Medicine and Science in Sports and Exercise 1986; 18: 501 - 508 .

24 Figoni SF. Exercise responses and quadriplegia. Medicine and Science in Sports and Exercise 1993; 25: $433-441$.

25 Davis GM et al. Cardiovascular responses to FNS-induced isometric leg exercise during lower body negative pressure. In: Proceedings of the 12th Annual RESNA Conference. New Orleans, Louisiana. 1989.

26 Figoni SF et al. Acute hemodynamic responses of spinal cord injured individuals to functional neuromuscular stimulationinduced knee extension exercise. Journal of Rehabilitation Research 1991; 28: 9-18.
27 Glaser RM. Spinal cord injury and neuromuscular stimulation. In: Current Therapy in Sports Medicine, Torg JS (ed). Decker, Incorporated Toronto, BC 1990, pp. 166-170

28 Figoni SF. Arm and leg exercise stress testing in a quadriplegic patient. Clinical Kinesiology (Abstract) 1989; 43: 108.

29 Burkett LN, Phillips WT, Paul JP. Arm crank exercise with static FNS in able-bodied persons. Sports Medicine, Training and Rehabilitation 1998; 7: $227-233$.

30 Eijsbouts XH, Hopman MTE, Skinner JS. EFfect of electrical stimulation of leg muscles on physiological responses during arm-cranking exercise in healthy men. European Journal of Applied Physiology 1997; 75: $177-181$

31 Miller VM, Aarhus LL, Vanhoutte PM. Modulation of endothelium-dependent responses by chronic alterations of blood flow. American Journal of Physiology 1986; 251: H520H527.

32 Langille BL, O'Donnell F. Reductions in arterial diameter produced by chronic decreases in blood flow are endotheliumdependent. Journal of Physiology (London) 1986; 108: 405-407.

33 Shenberger JS, Leaman GJ, Neumyer MM, Musch TI, Sinoway LI. Physiologic and structural indices of vascular function in paraplegics. Medicine and Science in Sports and Exercise, 1990; 22: $96-101$

34 United States Department of Health and Human Services, U. Healthy People 2000: National health promotion and disease prevention objectives. 1991, Public Health Service: Washington, DC.

35 Graciter PL, Maynard FM. eds. First Colloquium on Preventing Secondary Disabilities Among People with Spinal Cord Injuries. 1990, Centers for Disease Control: Atlanta, GA. 